\title{
Asociación de fractura de hioides con fractura panfacial
}

\author{
Association of hyoid fracture with pan-facial fracture
}

\author{
Ismael Vejar Alba, ${ }^{*}$ Guadalupe Jacinto Arias, ${ }^{\ddagger}$ Emmanuel Guerrero Soto ${ }^{\S}$
}

\section{RESUMEN}

Introducción: La fractura del hueso hioides es sumamente rara $(0.002 \%$ de todas las fracturas), puede presentar complicaciones como laceración faríngea, disnea, disfagia y pseudoaneurisma de la carótida externa, por lo que se debe realizar su pronta identificación y tratamiento. Se reporta el caso de un paciente de 23 años de edad que sufrió un accidente automovilístico durante actos del servicio, produciendo traumatismo cráneo encefálico moderado, neumotórax, fractura panfacial y fractura del hueso hioides. El paciente recibió un tratamiento inicial de urgencia consistente en colocación de sonda pleural y traqueostomía y al cuarto día del traumatismo se realizó tratamiento médico quirúrgico consistente en craneotomía descompresiva y cranealización de ambos senos frontales y reducción abierta más fijación interna con material de osteosíntesis de fracturas faciales. La fractura del hueso hioides se manejó de manera conservadora y actualmente el paciente se encuentra asintomático en relación con el habla, deglución y movimientos de hiperextensión cervical. Objetivo: Presentar el caso de una fractura del hueso hioides en relación con una fractura panfacial que se trató en el Hospital Central Militar. Diseño: Reporte de un caso clínico. Resultados: Reporte

\section{ABSTRACT}

Introduction: The fracture of the hyoid bone is extremely rare $(0.002 \%$ of all fractures), and may present complications such as pharyngeal laceration, dyspnea, dysphagia and pseudoaneurysm of the external carotid, so its prompt identification and treatment should be performed. The case of a 23-yearold patient who suffered a car accident during acts of the service, causing moderate cranioencephalic trauma, pneumothorax, panfacial fracture and hyoid bone fracture is reported. The patient received an initial emergency treatment consisting of placement of the pleural catheter and tracheostomy and on the 4th day of the trauma a surgical medical treatment was performed consisting of decompressive craniotomy and cranialization of both frontal sinuses and open reduction plus internal fixation with osteosynthesis material of facial fractures. The fracture of the hyoid bone was managed conservatively and currently the patient is asymptomatic in relation to speech, swallowing and cervical hyperextension movements. Objective: To present the case of a fracture of the hyoid bone in relation to a panfacial fracture that was treated at the Central Military Hospital. Design: Report of a clinical

* Jefe de la Subsección de Cirugía Oral y Maxilofacial del Hospital Central Militar, Jefe del Curso de Especialidad y Residencia en Cirugía Oral y Maxilofacial.

₹ Médico adscrito de la Subsección de Cirugía Oral y Maxilofacial del Hospital Central Militar. $\S$ Licenciado en Odontología, Residente de 20 año del Curso de Especialización y Residencia en Cirugía Oral y Maxilofacial.

Escuela Militar de Graduados de Sanidad. Hospital Central Militar. Ciudad de México, México.

Correspondencia:

Emmanuel Guerrero Soto

E-mail: guerrerosoto11@gmail.com

Citar como: Vejar AI, Jacinto AG, Guerrero SE. Asociación de fractura de hioides con fractura panfacial.

Rev Mex Cir Bucal Maxilofac. 2020; 16 (2 y 3): 97-102. https://dx.doi.org/10.35366/97707 
del trigésimo segundo caso de fractura del hueso hioides y el tercero en relación con una fractura panfacial.

Palabras clave: Fractura panfacial, hueso hioides, trauma, reducción. case. Results: Report of the 32nd case of fracture of the hyoid bone and the $3 r d$ in relation to a panfacial fracture.

Keywords: Panfacial fracture, hyoid bone, trauma, reduction.

\section{INTRODUCCIÓN}

El hioides en un hueso con forma de herradura que se compone de un cuerpo, dos astas mayores y dos astas menores. Está situado en la línea media superior a la laringe, a la altura de la cuarta vértebra cervical, mientras que la arteria carótida externa se encuentra profundamente al vientre posterior de los músculos digástrico y estilohioideo. En este hueso se insertan los músculos suprahioideos e infrahioideos, los cuales tienen como función general descender el hioides y la laringe, abatir la mandíbula y dar inserción a la lengua, laringe y faringe. Se encuentra aislado del resto del esqueleto facial, unido sólo por ligamentos y músculos, protegido anteriormente por el cuerpo mandibular y la musculatura cervical y posteriormente por las vértebras cervicales. ${ }^{1}$ Esta disposición anatómica hace que su fractura sea sumamente rara $\left(0.002 \%\right.$ de todas las fracturas). ${ }^{2,3}$ Las causas del trauma incluyen estrangulamiento, trauma contuso cervical, heridas por arma de fuego, uso de cascos con correa, hiperextensión cervical y vómito inducido. ${ }^{4,5}$ Su fractura se da en $48 \%$ de las veces en la unión del asta mayor con el cuerpo, en $49 \%$ en el asta mayor y en $3 \%$ en la parte media del cuerpo y ocupa una fuerza media de $30.55 \mathrm{~N} .{ }^{6}$ Un traumatismo de alto impacto como heridas por arma de fuego y accidentes automovilísticos producirá fracturas múltiples y con desplazamiento hacia fuera del hioides y en asociación con otras fracturas del esqueleto, a diferencia de cuando la etiología es el ahorcamiento o el estrangulamiento manual, donde sólo se ven fracturas aisladas y simples. ${ }^{7}$ El diagnóstico se basa en la sintomatología del paciente: dolor severo en el área de la faringe que se intensifica al tragar, toser o sonarse, disfagia y disnea. En la exploración física: inflamación, crepitación y sensibilidad a los movimientos de hiperextensión cervical. En los estudios complementarios: radiografías simples, tomografía computarizada (estándar de oro), laringoscopia y exploración quirúrgica en caso de trauma penetrante. Su tratamiento normalmente es conservador; sin embargo, se regirá a partir de las complicaciones que pueden llegar a acompañar su fractura, las cuales se pueden dividir en tempranas como el enfisema subcutáneo, disnea, laceración faríngea y del cartílago tiroideo; y tardías como disfagia, estridor y pseudoaneurisma de la arteria carótida externa. Se ha encontrado en relación con fracturas mandibulares, nasales, Le Fort III y fracturas panfaciales. ${ }^{8,9}$

Una fractura panfacial es aquélla que involucra el tercio superior, medio e inferior de la cara. ${ }^{10} \mathrm{Su}$ incidencia se encuentra entre 4 y $10 \%$ de todas las fracturas faciales. Normalmente es causada por traumatismos de alto impacto, tales como accidentes automovilísticos y heridas por armas de fuego. Hasta en $80 \%$ se encuentra en relación con fracturas condilares y en la mayoría de los casos tendrá segmentos conminutados y avulsionados. La tomografía computarizada es el estándar de oro para el diagnóstico en estudios de imagen. Se debe sospechar de lesiones cervicales y el mantenimiento de la vía aérea debe ser la prioridad. La traqueostomía se indica cuando es necesaria una intubación prolongada y cuando el complejo naso-órbito-etmoidal está involucrado, puesto que la intubación nasotraqueal sería difícil y peligrosa. ${ }^{11,12} \mathrm{El}$ orden de tratamiento para la reducción y fijación de las fracturas puede ser de arriba a abajo, de adentro hacia afuera, de abajo hacia arriba o de afuera hacia adentro. Sin embargo, de acuerdo con la AOCMF, el orden exacto de tratamiento no es tan importante como un adecuado plan que permita la posición anatómica correcta de los segmentos faciales tomando como base la exposición, identificación y fijación de los contrafuertes faciales. De manera que se devuelva la anchura, altura y proyección facial. ${ }^{13}$

\section{DESCRIPCIÓN DEL CASO}

Se reporta el caso de paciente masculino, militar en activo, de 23 años sin antecedentes personales y/o familiares de importancia, que sufre un accidente automovilístico tipo volcadura durante actos del servicio. Mientras se encontraba sentado en una camioneta de transporte militar, sin cinturón de seguridad y con el uso de casco y chaleco, el conductor perdió el control del vehículo, impulsándolo hacia adelante y golpeándose 
Figura 1:

Fotografía facial frontal a un día del traumatismo en la que se observa signo de ojos de mapache,

hundimiento

de pirámide nasal, distancia

intercantal

aumentada,

taponamiento

nasal anterior

y múltiples

abrasiones.
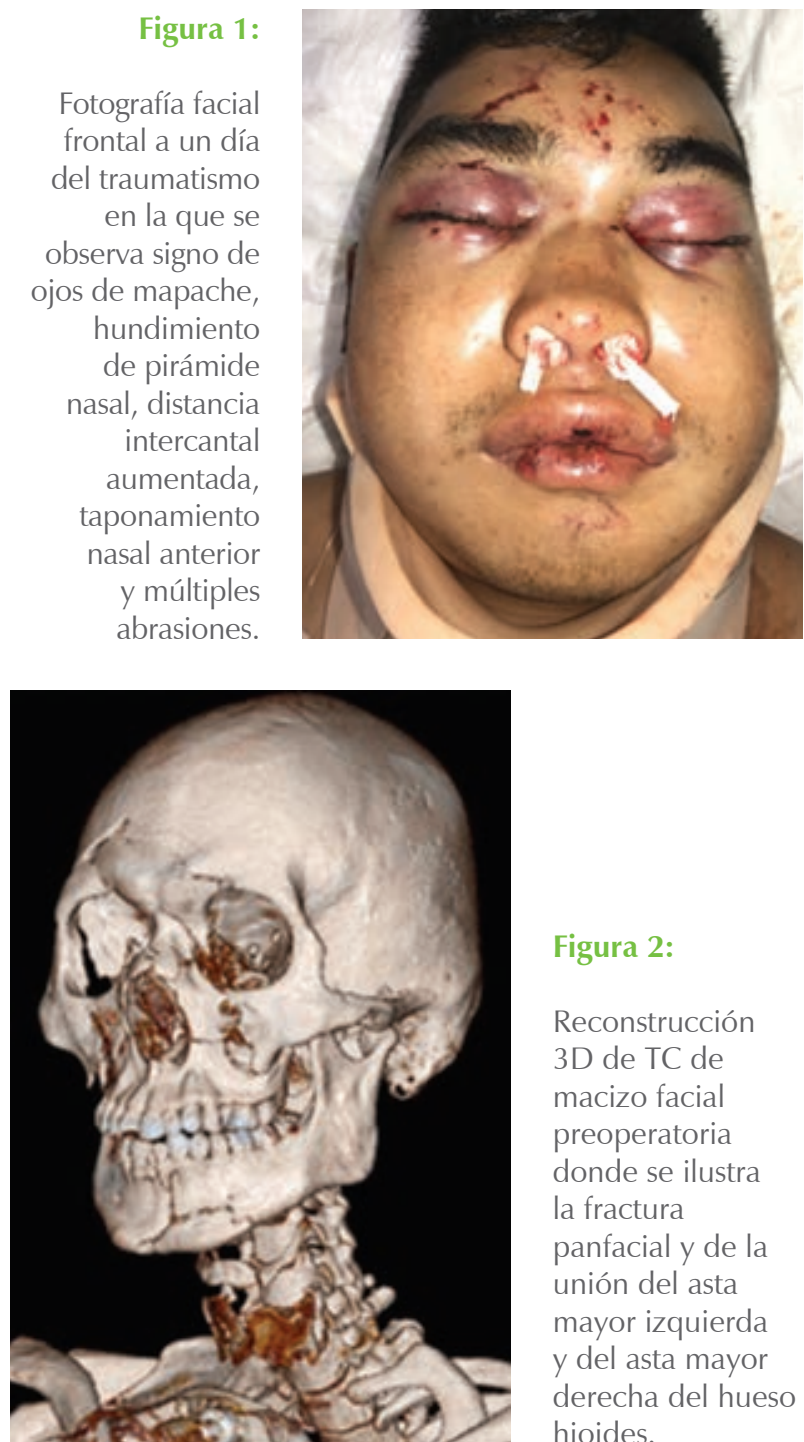

Figura 2:

Reconstrucción

3D de TC de

macizo facial

preoperatoria

donde se ilustra

la fractura

panfacial y de la

unión del asta

mayor izquierda

y del asta mayor

derecha del hueso

hioides.

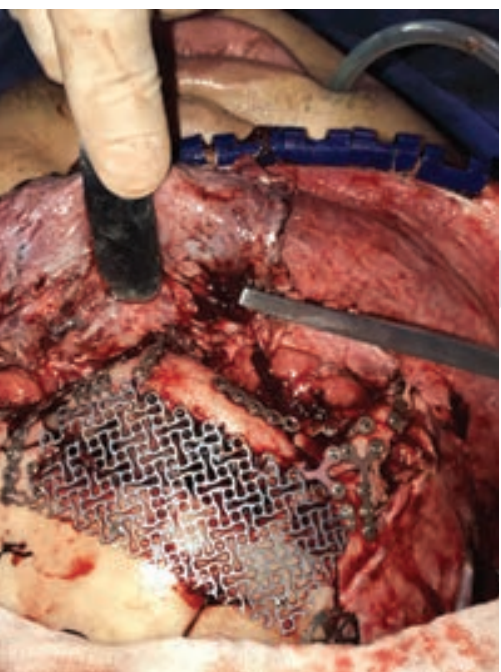
bicoronal. transoperatoria que muestra la reconstrucción e tercio medio superior a través de un abordaje
Figura 4:

Fotografía

transoperatoria

del abordaje

submentoniano

para la reducción

y fijación interna

de la fractura

sinfisiaria y del

borde basal.
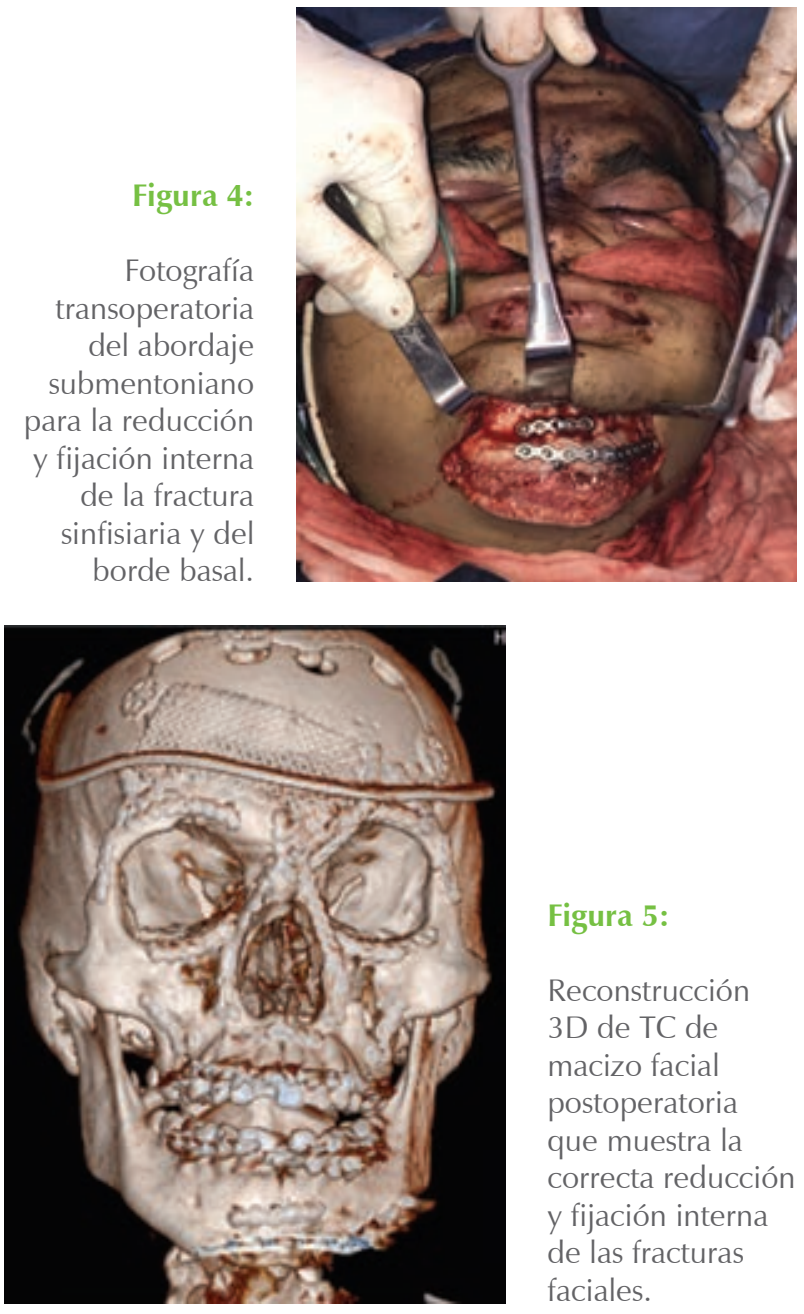

Figura 5:

Reconstrucción 3D de TC de macizo facial postoperatoria que muestra la correcta reducción y fijación interna de las fracturas faciales.

Figura 6:

Fotografía facial frontal del vigésimo día postoperatorio en la que se observa cicatriz de abordaje bicoronal, adecuada proporción de tercios medios faciales, adecuada distancia intercantal, abordajes subciliares en proceso de cicatrización y decanulación de tubo de traqueostomía.

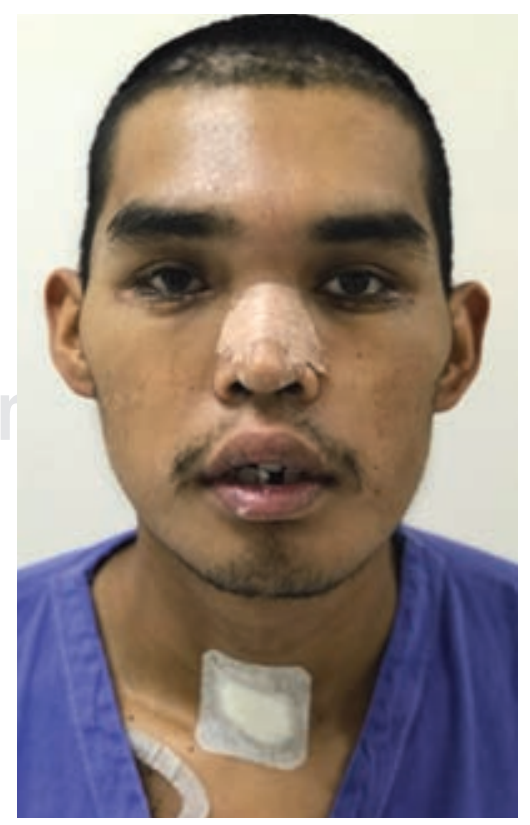


de manera frontal con el asiento tubular de metal de enfrente, golpe que produjo que perdiera la conciencia, posteriormente el vehículo cayó por un voladero, durante esta segunda volcadura fue impulsado fuera del vehículo cayendo sobre una superficie de piedras y pasto, recibió atención de primera instancia y fue trasladado en helicóptero al Hospital Militar Regional de Guadalajara, donde se determinó un diagnóstico de traumatismo cráneo-encefálico moderado, neumotórax y traumatismo facial y se decidió tratamiento consistente en colocación de sonda pleural y traqueostomía. Posteriormente fue transportado en helicóptero al Hospital Central Militar donde pasó directamente a la unidad de cuidados intensivos donde se sedó al paciente y se instauró ventilación mecánica, se colocó catéter venoso central a fin de administrar aminas tipo norepinefrina, cobertura antimicrobiana con cefalosporina de cuarta generación y glucopéptidos y colocación de sonda orogástrica (Figura 1). A la exploración clínica y al estudio de tomografía computarizada de macizo facial se dio un diagnóstico de fractura panfacial consistente en: fractura de pared anterior y posterior del seno frontal de manera bilateral, trazos de fractura Le Fort I, II y III, fractura NOE tipo II, fractura de piso, pared medial y lateral de ambas órbitas, fractura de arco cigomático derecho, fractura sagital palatina, fractura sinfisiaria y en bloque mandibular y fractura de la unión del asta mayor izquierda con el cuerpo y del asta mayor derecha del hueso hioides. Al cuarto día del traumatismo se realizó tratamiento médico quirúrgico consistente en craneotomía descompresiva y cranealización de ambos senos frontales por parte del Servicio de Neurocirugía, reducción abierta más fijación rígida con material de osteosíntesis de fractura panfacial + reconstrucción de órbitas con mallas de titanio + cantopexia del ligamento cantal derecho por parte del Servicio de Cirugía Oral y Maxilofacial del Hospital Central Militar. La fractura del hueso hioides se manejó de manera conservadora con traqueostomía, sonda orogástrica y collarín cervical (Figuras 2 a 4). Actualmente el paciente se encuentra ubicado en las tres esferas de conciencia, con movimientos oculares y agudeza visual conservada, oclusión estable, sin dificultad al habla y deglución, con valoración endoscópica por parte de la especialidad de foniatría, estado que le permitirá regresar a su servicio laboral sin restricción alguna (Figuras 5 y 6).

\section{Consideraciones éticas}

El paciente dio su consentimiento para la divulgación de su caso, y la toma y exposición de fotografías clínicas con fines académicos.

\section{DISCUSIÓN}

La fractura del hueso hioides es un evento sumamente raro, hasta el momento sólo se han reportado en la literatura 35 casos, siendo la causa más común los accidentes de tráfico en pacientes masculinos con una media de edad de 30 años y en la mayoría de los casos se presenta como una fractura aislada sin lesiones asociadas. La fractura panfacial sólo se ha encontrado en relación con dos casos (Tabla 1); sin embargo, sería justo pensar que existen muchas fracturas de hioides inadvertidas, lo cual puede obedecer a dos razones: la escasa atención que se da a esta estructura y la falta de conocimiento sobre las posibles complicaciones que puede llegar a producir su fractura.

Nuestro caso se encuentra dentro de esta prevalencia en cuanto a etiología, sexo y edad. El hallazgo es significativo al encontrarse en relación con fracturas de los tres tercios faciales, produciéndose una fractura múltiple característica de los traumatismos de alto impacto.

La clasificación de Weintraub de 1961 cataloga las fracturas del hueso hioides en:

1. Fracturas debido a compresión interna.

2. Fracturas debido a compresión anteroposterior.

3. Fracturas avulsivas.

Las fracturas por compresión interna producen desplazamiento del fragmento del asta mayor hacia el interior de éste, lo que coincide con las imágenes de nuestro caso. ${ }^{14}$

El tratamiento de las fracturas del hueso hioides se determina con base en las complicaciones que se presenten, en este sentido en la Tabla 2 se muestra la clasificación de estas complicaciones y su tratamiento propuesto con base en las referencias bibliográficas. Un seguimiento intrahospitalario de 48 a 72 horas es necesario aun en casos de pacientes asintomáticos a fin de descartar un cuadro de disnea.

Nuestro paciente fue tratado en la unidad de cuidados intensivos con sedación, ventilación mecánica por medio de traqueostomía, alimentación por sonda orogástrica y collarín rígido por el politraumatismo, con lo que se abarcó el tratamiento a fracturas del hueso hioides sin daños a estructuras adyacentes. A la evaluación secundaria por nuestro servicio, no presentó signos de laceración a estructuras adyacentes o bordes óseos que requirieran su regularización y/o fijación. En el periodo postoperatorio, el paciente 
Tabla 1: Casos reportados de fractura de hueso hioides.

\begin{tabular}{|c|c|c|c|c|c|}
\hline No. & Autor & $\begin{array}{l}\text { Edad } \\
\text { (años) }\end{array}$ & Sexo & Causa & Lesiones asociadas \\
\hline 1 & Guernsey (1945) & 45 & M & Accidente de tráfico & Fracturas mandibulares bilaterales \\
\hline 2 & Papavasiliou \& Speas (1959) & 23 & M & Herida por arma de fuego & Fractura mandibular \\
\hline 3 & Papavasiliou \& Speas (1959) & 22 & $\mathrm{~F}$ & Asalto & Fractura nasal \\
\hline 4 & Chadwick (1960) & 34 & M & Accidente de tráfico & Laceración faríngea \\
\hline 5 & Krekorian (1964) & 21 & M & Accidente de tráfico & Laceración faríngea \\
\hline 6 & Graf (1969) & 15 & M & Accidente de tráfico & Lesión espinal cervical \\
\hline 7 & Maran \& Stell (1970) & 22 & M & Deportes & Ninguna \\
\hline 8 & Browne (1973) & 16 & M & Asalto & Fracturas mandibulares bilaterales \\
\hline 9 & Eliachar et al. (1980) & 35 & M & Accidente de tráfico & $\begin{array}{l}\text { Fractura Le Fort III, fracturas mandibulares bilatera- } \\
\text { les, laceraciones faríngeas, lesión espinal cervical }\end{array}$ \\
\hline 10 & Eliachar et al. (1980) & 23 & $\mathrm{~F}$ & Accidente de tráfico & $\begin{array}{l}\text { Fracturas mandibulares bilaterales, laceraciones } \\
\text { faríngeas }\end{array}$ \\
\hline 11 & Eliachar et al. (1980) & 22 & M & Herida penetrante & Laceración faríngea \\
\hline 12 & Gregersen \& Vesterby (1981) & 53 & M & $\begin{array}{l}\text { Fractura iatrogénica: } \\
\text { resucitación }\end{array}$ & Fractura del cartílago tiroides \\
\hline 13 & Whyte (1985) & 29 & M & Accidente de tráfico & Fractura mandibular \\
\hline 14 & Zachariades (1985) & 34 & $M$ & Accidente de tráfico & Ninguna \\
\hline 15 & Zachariades \& Mezitis (1987) & 15 & $\mathrm{~F}$ & Accidente de tráfico & $\begin{array}{l}\text { Fractura Le Fort III, fracturas mandibulares múl- } \\
\text { tiples, fractura del hueso temporal, pérdida de } \\
\text { globo ocular }\end{array}$ \\
\hline 16 & Padgham (1988) & 15 & $M$ & Accidente de tráfico & Ninguna \\
\hline 17 & Szeremeta \& Morovati (1989) & 55 & M & Accidente de tráfico & Ninguna \\
\hline 18 & David \& Corrigan (1989) & 41 & M & Asalto & Laceración facial \\
\hline 19 & Dickenson (1991) & 25 & $M$ & Accidente industrial & Ninguna \\
\hline 20 & Lakhia et al. (1991) & 18 & $M$ & Desconocido & Ninguna \\
\hline 21 & Olu Ibekwe (1991) & 37 & $M$ & Accidente de tráfico & Laceración faríngea \\
\hline 22 & Carroll et al. (1992) & 23 & M & Herida por arma de fuego & Ninguna \\
\hline 23 & Gupta et al. (1995) & 28 & M & Vómito & Ninguna \\
\hline 24 & Kaufman et al. (1999) & 34 & M & Accidente de tráfico & $\begin{array}{l}\text { Laceración facial, fractura del cuerpo de la terce- } \\
\text { ra vértebra lumbar }\end{array}$ \\
\hline 25 & Kaufman et al. (1999) & 35 & M & Accidente de tráfico & Fracturas del tercio medio \\
\hline 26 & Kaufman et al. (1999) & 36 & M & Caída durante convulsión & Ninguna \\
\hline 27 & Doring \& Kahle (2000) & 17 & $M$ & Deportes & Ninguna \\
\hline 28 & Anthony et al. (2000) & 66 & M & $\begin{array}{l}\text { Posterior a cirugía de } \\
\text { cervicales }\end{array}$ & Ninguna \\
\hline 29 & Campbell et al. (2003) & 53 & $M$ & Desconocida & Pseudoaneurisma de la arteria carótida externa \\
\hline 30 & Dalati (2004) & 19 & M & Caída durante convulsión & Ninguna \\
\hline 31 & Dalati (2004) & 26 & M & Deportes & Ninguna \\
\hline 32 & Liang-Chi (2009) & 19 & M & Accidente de tráfico & Ninguna \\
\hline 33 & Carrillo (2009) & 15 & $\mathrm{~F}$ & Compresión cervical & Ninguna \\
\hline 34 & Yauh-Mirng (2012) & 36 & M & Caída de escalera & Laceración cervical \\
\hline 35 & Van Dongen (2016) & 62 & M & $\begin{array}{l}\text { Caída de bicicleta con el } \\
\text { uso de casco de correa }\end{array}$ & Fractura Le Fort III, laceración facial \\
\hline 36 & Vejar, Jacinto \& Guerrero (2019) & 23 & M & Accidente de tráfico & $\begin{array}{l}\text { Fractura de pared anterior y posterior del seno } \\
\text { frontal de manera bilateral, fractura Le Fort III y } \\
\text { múltiples fracturas mandibulares }\end{array}$ \\
\hline
\end{tabular}


Rev Mex Cir Bucal Maxilofac 2020;16 (2 y 3): 97-102

Tabla 2: Clasificación de complicaciones de fractura de hueso hioides y su tratamiento propuesto.

\begin{tabular}{|c|c|c|c|c|}
\hline $\begin{array}{l}\text { Paciente } \\
\text { asintomático }\end{array}$ & $\begin{array}{l}\text { Dolor de moderado } \\
\text { a severo sin lesión a } \\
\text { estructuras anatómicas } \\
\text { adyacentes }\end{array}$ & Laceración faríngea & $\begin{array}{l}\text { Laceración externa del } \\
\text { cuello }\end{array}$ & Dificultad respiratoria \\
\hline $\begin{array}{l}\text { - No necesita } \\
\text { tratamiento } \\
\text { - Vigilancia por } \\
\text { 48-72 horas }\end{array}$ & $\begin{array}{l}\text { - Analgésicos orales } \\
\text { - Alimentación vía sonda } \\
\text { nasogástrica } \\
\text { - Movimiento restringido } \\
\text { - Valoración por foniatra } \\
\text { - Seguimiento intrahospi- } \\
\text { talario de 48-72 horas }\end{array}$ & $\begin{array}{l}\text { - Sutura de heridas profundas } \\
\text { - Remover fragmentos óseos } \\
\text { del hueso hioides o reduc- } \\
\text { ción de los mismos con } \\
\text { alambre } \\
\text { - Regularizar los bordes } \\
\text { - Alimentación por tubo } \\
\text { gástrico }\end{array}$ & $\begin{array}{l}\text { - Remoción de fragmen- } \\
\text { tos óseos } \\
\text { - Sutura de heridas } \\
\text { superficiales }\end{array}$ & $\begin{array}{l}\text { - Intubación endotraqueal o } \\
\text { traqueostomía } \\
\text { - Exposición quirúrgica y } \\
\text { drenaje del espacio retro- } \\
\text { faríngeo en caso de enfise- } \\
\text { ma subcutáneo severo }\end{array}$ \\
\hline
\end{tabular}

no presentó signos de dificultad respiratoria y tras la prueba de deglución por el servicio de foniatría se autorizó el retiro de la sonda orogástrica y decanulación de traqueostomía, descartando cualquier posible complicación de la fractura del hioides.

\section{CONCLUSIÓN}

Reportamos el trigésimo sexto caso de fractura del hueso hioides y el tercero en relación con una fractura panfacial. Al conocer las posibles complicaciones que pueden poner en riesgo la vida del paciente, se debe prestar más atención al diagnóstico y tratamiento de esta fractura.

\section{BIBLIOGRAFÍA}

1. Rouviere H, Delmas A. Anatomía humana: descriptiva, topográfica y funcional. 11a edición. México, D.F.: Masson; 2005.

2. Carrillo RJ, Mastranzo CH, Sánchez PV. Fractura de hueso hioides. Caso clínico y revisión bibliográfica. Rev Mex Cir Bucal Maxilofac. 2009; 5 (2): 68-71.

3. van Dongen MJ, Falger-Veeken SN. The risk of a bicycle helmet: hyoid bone fracture. Ann Emerg Med. 2016; 67 (1): 145-146.

4. Jehng YM, Lee FTT, Pai YC, Choi WM. Hyoid bone fracture caused by blunt neck trauma. J Acute Med. 2012; 2 (3): 83-84.
5. Kuo LC, Lin HL, Chen CW, Lee WC. Traumatic hyoid bone fracture in patient wearing a helmet: a case report. Am J Emerg Med. 2008; 26 (2): 251.e1-2.

6. Lebreton-Chakour C, Godio-Raboutet Y, Torrents R, Chaumoitre $\mathrm{K}$, Boval C, Bartoli $\mathrm{C}$ et al. Manual strangulation: experimental approach to the genesis of hyoid bone fractures. Forensic Sci Int. 2013; 228 (1-3): 47-51.

7. Weintraub CM. Fractures of the hyoid bone. Med Leg J. 1961; 29: 209-216.

8. Dalati T. Isolated hyoid bone fracture. Review of an unusual entity. Int J Oral Maxillofac Surg. 2005; 34: 449-452.

9. Whyte M. Fracture of the hyoid bone associated with a mandibular fracture. J Oral Maxillofac Surg. 1985; 43: 805-807.

10. Yang R, Zhang C, Liu Y, Li Z, Li Z. Why should we start from mandibular fractures in the treatment of panfacial fractures? J Oral Maxillofac Surg. 2012; 70 (6): 1386-1392.

11. Curtis W, Horswell BB. Panfacial fractures: an approach to management. Oral Maxillofac Surg Clin North Am. 2013; 25 (4): 649-660.

12. Miloro M, Ghali GE, Larsen PE, Waite PD. Peterson's principles of oral and maxillofacial surgery. 3rd ed. China: People's Medical Publishing House; 2011.

13. Ehrenfeld M, Manson PN, Prein J. Principles of internal fixation of the craniomaxillofacial skeleton trauma and orthognathic surgery. Switzerland: Georg Thieme Verlag; 2012.

14. Keerthi R, Quadri A. Hyoid bone fracture: associated with head and neck trauma-a rare case report. J Maxillofac Oral Surg. 2016; 15 (Suppl 2): 249-252.

Conflicto de intereses: No hay conflicto de intereses. 